\title{
Erfolgreiche Behandlung von Pemphigus foliaceus und ankylosierender Spondylitis mit Infliximab und Methotrexat: ein Fallbericht
}

\author{
Hilal Kaya Erdogan ${ }^{\mathrm{a}}$ Nihan Yuksel Canakci ${ }^{\mathrm{a}}$ Ersoy Acer ${ }^{\mathrm{a}} \quad$ Cengiz Korkmaz $^{\mathrm{b}}$ Evrim Yılmaz \\ Zeynep Nurhan Saracoglu ${ }^{a}$ \\ aDepartment of Dermatology, Eskişehir Osmangazi University Faculty of Medicine, Eskisehir, Türkei; \\ ${ }^{b}$ Department of Internal Medicine, Division of Rheumatology, Eskişehir Osmangazi University Faculty of Medicine, Eskisehir, Türkei; \\ 'Department of Pathology, Eskişehir Osmangazi University Faculty of Medicine, Eskisehir, Türkei
}

Sehr geehrter Herausgeber,

Pemphigus foliaceus (PF) ist eine chronische, blasenbildende $\mathrm{Au}$ toimmunerkrankung, die durch Immunglobulin-G (IgG)-Autoantikörper gegen Desmoglein 1 gekennzeichnet ist [1]. In der vorliegenden Arbeit stellen wir den Fall eines Patienten mit PF vor, der gleichzeitig eine ankylosierende Spondylitis (AS) hatte und erfolgreich mit Infliximab und Methotrexat behandelt wurde.

Ein 41-jähriger Mann stellte sich mit seit 3 Monaten bestehenden juckenden bullösen Eruptionen vor, die sich zu oberflächlichen Erosionen entwickelten. Bei der dermatologischen Untersuchung fanden sich weißlich-gelbe, verkrustete, oberflächliche Erosionen auf erythematösem Grund im Gesicht, auf der Kopfhaut, am Oberkörper sowie an den Oberschenkeln und Oberarmen (Abb. 1a). Er hatte seit 1 Jahr AS und erhielt deswegen Indomethacin. Die histopathologische Untersuchung der Stanzbiopsie ergab eine subkorneale vesikuläre Dermatitis (Abb. 2) und in der direkten Immunfluoreszenzuntersuchung wurde eine intraepidermale interzelluläre Färbung mit IgG und Komplement 3 nachgewiesen. Die Diagnose lautete Pemphigus foliaceus und der Patient erhielt Methylprednisolon $60 \mathrm{mg} / \mathrm{Tag}$ sowie Azathioprin $100 \mathrm{mg} /$ Tag. Die Dosierung von Methylprednisolon wurde über einen Zeitraum von 6 Monaten gesenkt und schließlich abgesetzt, woraufhin die PF-Läsionen innerhalb kurzer Zeit erneut auftraten. Wir setzten Azathioprin ab und begannen eine Behandlung mit Mycophenolat-Mofetil $2 \mathrm{~g}$ täglich. Nach 18-monatiger Behandlung mit Mycophenolat-Mofetil kam es zu einem erneuten Auftreten der Läsionen. Der Patient wurde der rheumatologischen Abteilung vorgestellt, wo eine schwere AS (Sakroiliitis im Stadium 4) und eine starke Einschränkung der Gelenkbeweglichkeit festgestellt wurde. In Absprache zwischen der rheumatologischen und der dermatologischen Abteilung wurde eine Behandlung mit
Infliximab $5 \mathrm{mg} / \mathrm{kg}$ und Methotrexat $15 \mathrm{mg} /$ Woche eingeleitet. Mycophenolat-Mofetil wurde abgesetzt und gemäß den Leitlinien zur Behandlung der AS erhielt der Patient Infliximab an Tag 0 , Tag 15, in Woche 6 und danach alle 8 Wochen. Nach 6 Therapiezyklen mit Infliximab waren die PF-Läsionen rückläufig (Abb. 1b) und die Gelenkbeweglichkeit hatte sich gebessert. Nach Abheilung der Läsionen, und als keine neuen Läsionen mehr auftraten, wurde Methotrexat ausgeschlichen und abgesetzt (kumulative Dosis: $375 \mathrm{mg}$ ). Die Behandlung mit Infliximab (insgesamt 8 Zyklen) hatte keine Nebenwirkungen und der Patient wird weiterhin beobachtet.

Man nahm an, dass bei der Pathogenese des Pemphigus entzündliche Zytokine eine Rolle spielen [1]. Feliciani et al. [2] fanden hohe Spiegel von Interleukin (IL)1- $\alpha$ und Tumornekrosefaktor (TNF)- $\alpha$ und eine verminderte Akantholyse unter Anti-IL1- $\alpha$ und Anti-TNF- $\alpha$-Antikörpern [2]. Lopez-Robles et al. [3] stellten fest, dass Biopsien aus den Läsionen von Pemphigus-Patienten eine höhere Zytokin-Genexpression und stärkere Ablagerungen von TNF- $\alpha$ und IL- 6 zeigten als nichtläsionale Biopsien. Zudem stellten sie fest, dass PF-Biopsien eine stärkere TNF- $\alpha$ - und IL6-Expression aufwiesen als bei anderen Pemphigustypen [3]. Über die Behandlung von Pemphigus mit Anti-TNF-Wirkstoffen liegen zahlreiche Berichte vor. Pardo et al. [1] berichteten über eine komplette Remission unter Infliximab bei einem Patienten mit Pemphigus vulgaris (PV), der gegen verschiedene Immunsuppressiva resistent war. Jacobi et al. [4] beobachteten ebenfalls ein rasches Ansprechen auf Infliximab bei einem PV-Patienten mit Therapieresistenz gegenüber anderen Immunsuppressiva. Sie schlugen vor, dass Infliximab eingesetzt werden kann, um eine schnelle PV-Kontrolle zu erzielen oder das Ansprechen auf eine immunsuppressive Standardtherapie wiederherzustellen. 

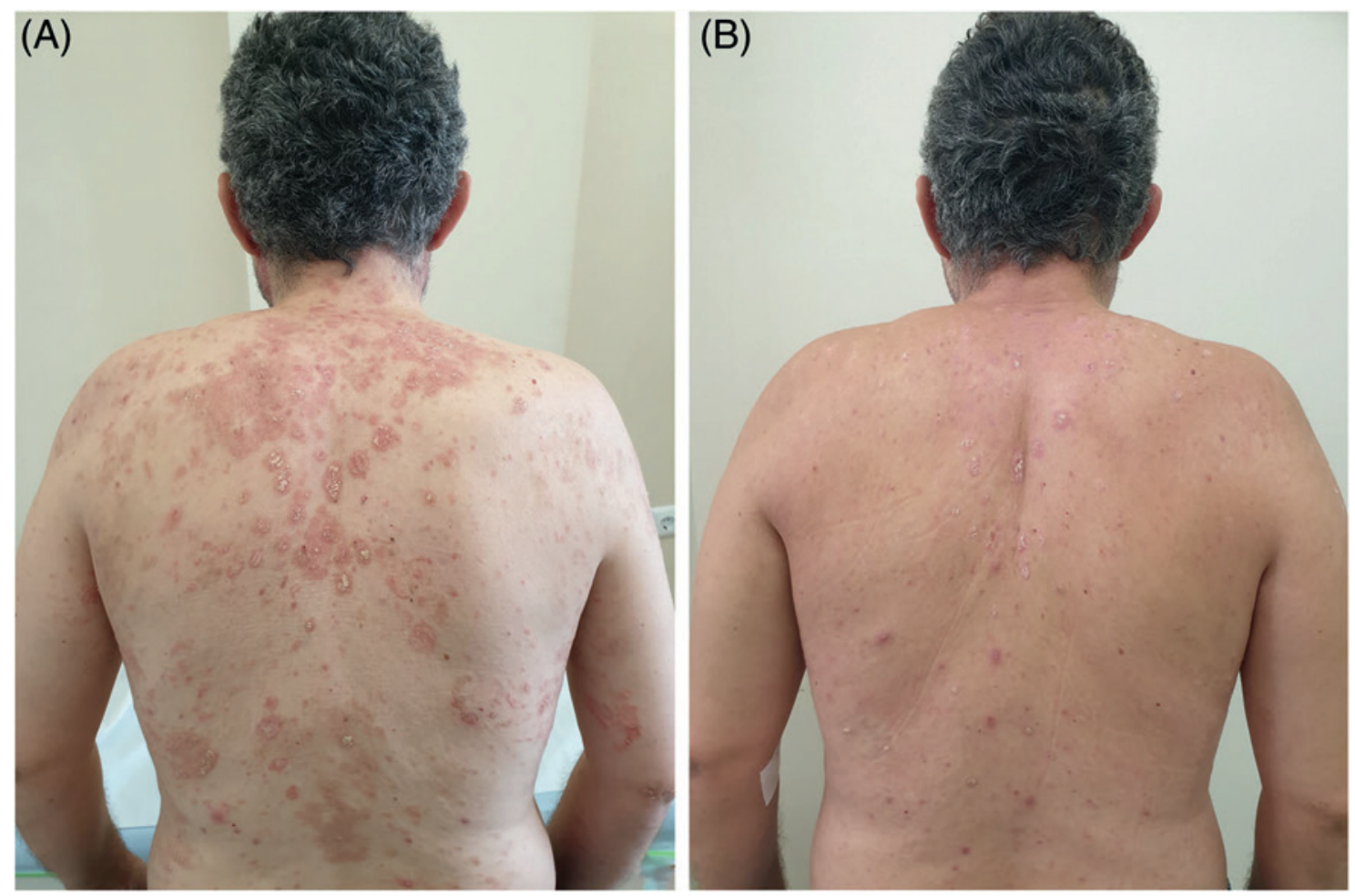

Abb. 1. (a) Generalisierte weißlich-gelbe, verkrustete, oberflächliche Erosionen auf erythematösem Grund (vor der Behandlung). (b) Rückbildung der Läsionen nach 6 Therapiezyklen mit Infliximab.
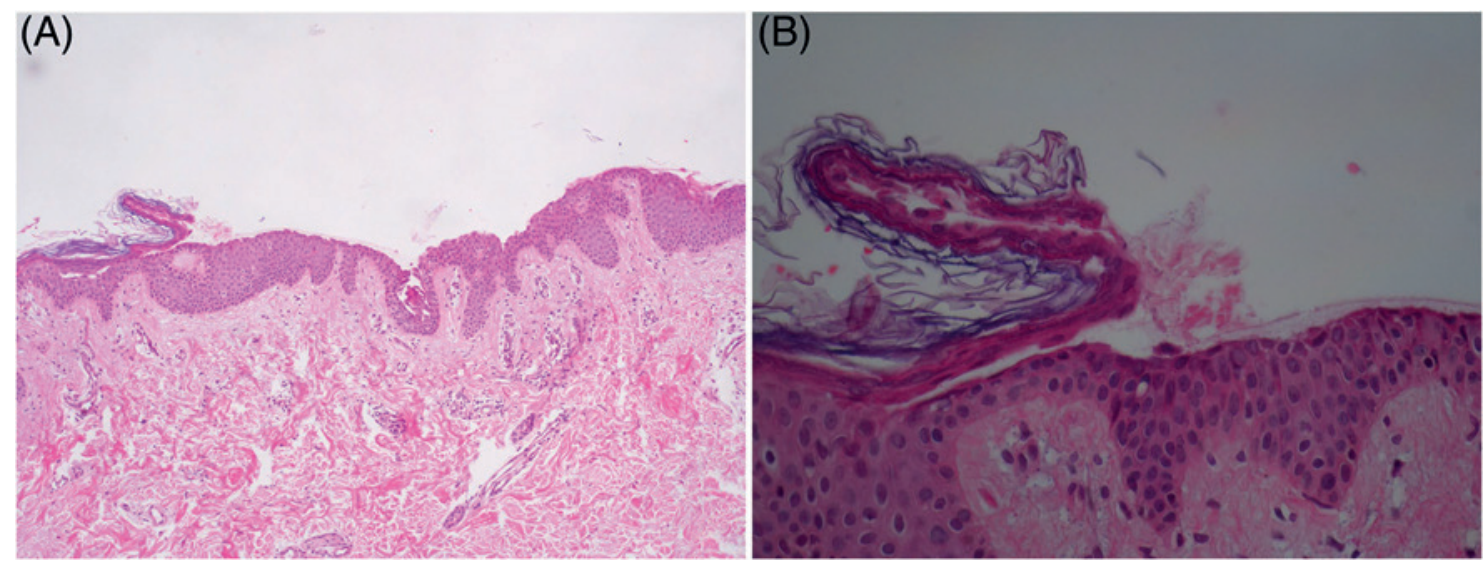

Abb. 2. Oberflächliche Ablösung der Epidermis. (a) Hämatoxylin-Eosin (H\&E), 10-fach; (b) H\&E, 40-fach.

Gubinelli et al. [5] berichteten über eine komplette Remission unter der Behandlung mit Etanercept und Methylprednisolon bei einem Patienten mit PV. Dagegen erzielten García-Rabasco et al. [6] mit Infliximab bei 2 therapieresistenten PV-Patienten eine kurzfristige initiale Remission; im weiteren Verlauf mussten sie jedoch Infliximab absetzen, da es unter der Behandlung zu einer Exazerbation der Läsionen kam. Ebenso fand eine Studie keine Wirksamkeit durch die Zugabe von Infliximab zu Prednisolon bei der Behandlung von PV [7]. Methotrexat erwies sich ebenfalls als wirksam bei der Behandlung von PV [8].

Bei unserem Patienten wurde 1 Jahr vor dem Auftreten der PFLäsionen eine AS diagnostiziert. Er nahm nur Indomethacin ein und entwickelte eine deutliche Bewegungseinschränkung. Der Patient wurde der rheumatologischen Abteilung vorgestellt, wo eine AS im fortgeschrittenen Stadium festgestellt wurde. In Absprache zwischen den beiden Abteilungen entschied man, eine Infliximab-Therapie zu beginnen, um beide Erkrankungen $\mathrm{zu}$ behandeln. Initial wurde Infliximab zusammen mit Methotrexat gegeben; nach Abheilung der Läsionen, und als keine neuen Läsionen mehr auftraten, wurde Methotrexat ausgeschlichen und abgesetzt. Unter der Kombination von Biologika und systemisch wirksamen Arzneimitteln können Nebenwirkungen wie Immunsuppression und ein erhöhtes Infektionsrisiko auftreten; bei unserem Patienten waren jedoch keine Nebenwirkungen zu beob- 
achten. Die Behandlung mit Infliximab wird fortgesetzt und ist mit einer deutlichen Verbesserung der Gelenkbeweglichkeit verbunden; neue Läsionen wurden nicht festgestellt.

Bei der AS handelt es sich um eine entzündliche Erkrankung, die vor allem das Achsenskelett betrifft. Chen et al. [9] stellten fest, dass das Risiko für anteriore Uveitis, Psoriasis, Thromboangiitis obliterans, Morbus Behçet und Sarkoidose bei AS-Patienten signifikant erhöht war, wohingegen beim Pemphigus-Risiko kein signifikanter Unterschied bestand. Es liegen in der Literatur keine Berichte vor, die einen Zusammenhang zwischen AS und Pemphigus belegen. Ob dem gleichzeitigen Auftreten von PF und AS bei unserem Patienten ein Zusammenhang zugrunde liegt oder ob es sich um reinen Zufall handelt, ist nicht klar.

Insgesamt spricht unser Bericht dafür, dass Infliximab bei der Behandlung des therapieresistenten PF wirksam sein kann. Un- seres Wissens ist dies der erste Fall von gleichzeitig bestehendem PF und AS, der erfolgreich mit Infliximab und Methotrexat behandelt wurde. Weitere Berichte sind erforderlich, um die Rolle von TNF- $\alpha$ in der Ätiopathogenese von PF und die Wirksamkeit von Anti-TNF-Therapien zu verstehen.

\section{Lizenzangabe}

Kaya Erdogan H, Yuksel Canakci N, Acer E, Korkmaz C, Yılmaz E, Saracoglu ZN. Successful treatment of pemphigus foliaceous and ankylosing spondylitis with infliximab and methotrexate: A case report. Dermatol Ther. 2020 Jul;33(4):e13801 (DOI: 10.1111/dth.13801). ${ }^{\circledR} 2020$ Wiley Periodicals LLC, (Übersetzung), lizensiert unter CC BY 4.0 (https://creativecommons.org/licenses/by/4.0/deed.de).

\section{Literatur}

Die Literatur ist unter www.karger.com/doi/10.1159/000518360 abrufbar. 